\title{
CT Hounsfield Units of Brown Adipose Tissue Increase with Activation: Preclinical and Clinical Studies
}

\author{
Shingo Baba ${ }^{1,2}$, Heather A. Jacene ${ }^{1}$, James M. Engles ${ }^{1}$, Hiroshi Honda ${ }^{2}$, and Richard L. Wahl ${ }^{1}$ \\ ${ }^{I}$ Division of Nuclear Medicine, Russell H. Morgan Department of Radiology and Radiological Science, Johns Hopkins Medical \\ Institutions, Baltimore, Maryland; and ${ }^{2}$ Department of Clinical Radiology, Graduate School of Medical Sciences, Kyushu \\ University, Fukuoka, Japan
}

\begin{abstract}
Brown adipose tissue (BAT) densities assessed as CT Hounsfield units (HUs) were evaluated in a rodent model and in patients to determine whether HUs changed in relation to BAT activity. Methods: Serial ${ }^{18} \mathrm{~F}-\mathrm{FDG}$ PET/CT was performed on rats under both room temperature control conditions and after $4 \mathrm{~h}$ of coldstimulation, which is known to activate BAT. The maximum standardized uptake values and CT HUs of BAT were measured, and tissues were examined in the laboratory. Image records from cancer patients who underwent PET/CT were reviewed, and 23 patients were identified who displayed both high and low ${ }^{18} \mathrm{~F}$ FDG uptake into BAT on serial ${ }^{18} \mathrm{~F}-\mathrm{FDG}$ PET/CT scans. The maximum standardized uptake values and CT HUs of BAT were compared in these scans. Results: The mean ( \pm SD) CT HUs of cold-activated BAT $(-12.4 \pm 22.4)$ were significantly higher than those $(-27.9 \pm 9.6)$ of the controls in the rat model. The CT HUs of BAT $(-71.6 \pm 18.0)$ in the patients with high ${ }^{18} \mathrm{~F}-\mathrm{FDG}$ uptake were significantly higher than those $(-104.4 \pm 16.8)$ of the patients with low ${ }^{18} \mathrm{~F}-\mathrm{FDG}$ uptake. A decrease in relative lipid content is seen in activated BAT in rats on histology. Conclusion: The CT HUs of BAT increased in activated conditions in both animals and patients, likely because of lipid consumption by activated BAT.
\end{abstract}

Key Words: BAT; CT Hounsfield unit; FDG; physiological uptake

J Nucl Med 2010; 51:246-250

DOI: 10.2967/jnumed.109.068775

B rown adipose tissue (BAT) is the main organ involved in nonshivering heat production and is regarded as important for the maintenance of the body's energy metabolism $(1,2)$. Activation of BAT is dynamically regulated via the sympathetic nervous system in response to the signals of decreasing body temperature, recent feeding, or high body energy reserve. BAT differs from white adipose tissue (WAT) morphologically. BAT is so named because of its brownish

Received Jul. 25, 2009; revision accepted Oct. 7, 2009.

For correspondence or reprints contact: Richard L. Wahl, Division of Nuclear Medicine, Department of Radiology, Johns Hopkins Medical Institutions, 601 N. Caroline St., Room 3223, Baltimore, MD 21287-0817. E-mail: rwahl@jumi.edu

COPYRIGHT @ 2010 by the Society of Nuclear Medicine, Inc. color on visual assessment, which results from its rich vascularization and increased mitochondrial content.

BAT is also important in clinical nuclear medicine, especially in oncologic diagnosis using PET with ${ }^{18} \mathrm{~F}-$ FDG. Nonmalignant, high-level uptake of ${ }^{18}$ F-FDG in the supraclavicular area, thoracic costovertebral regions, and other regions of the upper abdomen has been shown to primarily reflect uptake in BAT $(2,3)$. Increased uptake in the supraclavicular region has been reported to occur in $2.5 \%-4.0 \%$ of patients undergoing ${ }^{18} \mathrm{~F}$-FDG PET/CT (3). Such BAT uptake is more common in women and appeared to be more common in winter in several studies (3-6). The high uptake of ${ }^{18} \mathrm{~F}-\mathrm{FDG}$ in activated BAT may sometimes be indistinguishable from uptake in tumors or lymph nodes. Tumor or metastatic lymph node ${ }^{18}$ F-FDG uptake may be masked by nearby high ${ }^{18} \mathrm{~F}$-FDG uptake in BAT, potentially leading to false-negative or false-positive interpretations and errors in patient management.

Shortly after the introduction of PET/CT fusion technology, it was recognized that this focal ${ }^{18} \mathrm{~F}-\mathrm{FDG}$ uptake was occurring in BAT rather than in muscle $(3,4)$. This discovery was based mainly on CT findings, and particularly on Hounsfield units (HUs). Active BAT can be identified when the ${ }^{18} \mathrm{~F}-\mathrm{FDG}$-avid foci have low CT HUs (i.e., fat tissue) in a fusion image with PET/CT. When high ${ }^{18} \mathrm{~F}-\mathrm{FDG}$ uptake in BAT is unilateral or at an unusual site, it is extremely difficult to identify the BAT only by PET findings, and thus it is important to understand the CT findings of BAT $(7,8)$. To our knowledge, there has been no report describing BAT based solely on CT findings.

BAT is known to be activated through physiologic or pharmacologic intervention, and this activation results in an increase in ${ }^{18} \mathrm{~F}-\mathrm{FDG}$ uptake (9). We previously reported that the uptake of ${ }^{18} \mathrm{~F}-\mathrm{FDG}$ in BAT could be increased by a short period of cold-exposure in animal models (9), and recently this ability was also confirmed with human studies (10). Significant variation in ${ }^{18} \mathrm{~F}-\mathrm{FDG}$ uptake in BAT within individual patients has been reported. Christensen et al. reported patients who displayed a complete reversal of high BAT tracer uptake after simple core-warming maneuvers 
(11). In our previous rodent study, we observed a significant decrease in the lipid content of BAT tissue in rats after a short interval of cold-activation (12). These results suggest that the contents of BAT (both pathologically and chemically) would also be dynamically changed between an activated state and a nonactivated state in humans.

Based on the evidence that high ${ }^{18} \mathrm{~F}-\mathrm{FDG}$ uptake in BAT indicates BAT has been activated, we hypothesized that the CT HUs of activated BAT would be higher than those in nonactivated BAT because of the decreased lipid content. To test this hypothesis, we performed serial ${ }^{18} \mathrm{~F}$-FDG PET/ CT of BAT in rats under both control and cold-activated conditions and compared the ${ }^{18} \mathrm{~F}-\mathrm{FDG}$ uptake and CT HUs, as well as BAT histology. We also selected patients who had undergone serial ${ }^{18}$ F-FDG PET/CT and who had displayed varying degrees of BAT uptake (high and low) at varying time points on their scans. We then compared their standardized uptake values (SUVs) and CT HUs of BAT.

\section{MATERIALS AND METHODS}

\section{Animal Study}

Animals. Eight- to 12-wk-old female Lewis rats $(n=6$; mean body weight, $212 \mathrm{~g}$ ) were used in this study. Two serial ${ }^{18} \mathrm{~F}-\mathrm{FDG}$ PET/CT scans (day 1 and day 3) were obtained under different conditions (control and cold-stimulation) using the same set of animals. In the control condition (day 1), animals were kept at room temperature (approximately $23-24^{\circ} \mathrm{C}$ ) before ${ }^{18} \mathrm{~F}$-FDG injection. To stimulate BAT activity, the same experimental animals were maintained at $4^{\circ} \mathrm{C}$ for $4 \mathrm{~h}$ before tracer injection (day 3 ). Animals were held in a hand-made housing unit, and we obtained the CT scan immediately after the PET scan without changing the position of the animal. This method enabled precise fusion of the small-animal PET and CT images taken by the 2 scanners. The study was performed as part of a project that has been approved by the animal research committee at the Johns Hopkins Medical Institutions.

Small-Animal Imaging (PET and CT). PET images of animals were acquired for $15 \mathrm{~min}$ ( 3 bed positions) at $1 \mathrm{~h}$ after intravenous injection of $37 \mathrm{MBq}$ of ${ }^{18} \mathrm{~F}-\mathrm{FDG}$ (days 1 and 3) using a dedicated small-animal PET scanner (eXplore VISTA; GE Healthcare). Images were reconstructed using Fourier rebinning with 2dimensional ordered-subsets expectation maximization ( 2 iterations, 16 subsets).

CT images were acquired using the $\mathrm{CT}$ portion of a smallanimal SPECT/CT scanner (X-SPECT; Gamma Medica). Highresolution CT images were obtained at $50 \mathrm{kVp}$ and $0.6 \mathrm{~mA}$. Images were captured for $2.5 \mathrm{~s}$ per view for 256 views in a $360^{\circ}$ rotation. The animals were kept anesthetized using isoflurane gas during the PET and CT acquisitions.

\section{Ex Vivo Study}

BAT specimens with an adequate amount of surrounding subcutaneous WAT were resected from euthanized animals (this set of animals was different from that used in the in vivo imaging study) kept under 2 different conditions (control and cold-stimulation, as in the in vivo study). The specimens were collectively added to a 50-mL tube filled with water, and CT images of the specimens were acquired using the X-SPECT scanner and the same parameters as for the in vivo animal study. To visualize the histologic changes of BAT, we stained samples from the control and coldstimulated animals with hematoxylin and eosin. The samples were observed with a light microscope.

\section{Patient Study}

Patient Population. We retrospectively reviewed the reports of 1,519 consecutive whole-body PET/CT scans (1,384 patients), which were performed for various clinical indications with followup period between 2001 and 2007. Of those patients, 904 are from Johns Hopkins University Hospital and 480 are from Kyushu University Hospital. We identified 73 patients who displayed high ${ }^{18}$ F-FDG uptake in BAT. Among them, 23 patients were selected (Table 1) who met the following entry criteria: receipt of at least 2 PET/CT scans, high BAT uptake (maximum SUV [SUV max $>3$ ) in 1 study and low uptake $\left(\mathrm{SUV}_{\max }<2\right)$ in another study, no gross lesions or abnormalities in the supraclavicular region, and no significant change in body weight between the 2 studies (change of body mass index $<5 \mathrm{~kg} / \mathrm{m}^{2}$ ). The final selected patients had no evidence of previous BAT-altering medication such as $\beta$-blockers and no history of diabetes mellitus or hypermetabolic disease such as pheochromocytoma. Our institutional review boards approved this study under an expedited review and waived the requirement for informed consent.

PET/CT Scans. All patients were imaged using our standard clinical PET/CT protocol. Patients fasted for at least 4-6 h before the PET acquisition. Blood glucose, which had to be less than 7 $\mathrm{mmol} / \mathrm{L}$, was checked before injection of $310-651 \mathrm{MBq}$ of ${ }^{18} \mathrm{~F}$ FDG. Intravenous injection was followed by a tracer uptake period of about $60 \mathrm{~min}$. No muscle relaxants were administered. Patients were asked to breathe normally during PET and CT acquisitions. Scanning was performed using a Discovery LS or STE PET/CT scanner (GE Healthcare). PET emission data were acquired in 2-dimensional mode for $5 \mathrm{~min} / \mathrm{bed}$ position.

\section{Image Analysis}

For the animal study, PET data were converted to an interfile format and analyzed using ImageJ software (http://rsbweb.nih.gov/ $\mathrm{ij} /$ ). ${ }^{18} \mathrm{~F}-\mathrm{FDG}$ uptake in BAT was assessed as counts/s/mL of tissue using a manually placed region of interest (ROI). CT HUs of BAT were calculated manually using linear interpolation of water CT density (assume CT HU = 0) and air CT density (assume CT HU = $-1,000)$ with ImageJ software. Fusion images of PET and CT were made using AMIDE software (http://amide.sourceforge.net/).

For the human study, PET images were analyzed on a Xeleris workstation (GE Healthcare). In this study, high ${ }^{18}$ F-FDG uptake

\begin{tabular}{lc}
\hline $\begin{array}{l}\text { TABLE 1. Patient and Disease Characteristics } \\
\text { (BAT-Avid Cohort) }\end{array}$ \\
\hline \multicolumn{1}{c}{ Characteristic } & Value \\
Age $(\mathrm{y})$ & \\
Mean \pm SD & $34 \pm 17$ \\
Range & $17-54$ \\
Men/women $(n)$ & $5 / 18$ \\
Disease $(n)$ & \\
Ovarian carcinoma & 5 \\
Non-Hodgkin lymphoma & 3 \\
Hodgkin disease & 2 \\
Thyroid carcinoma & 3 \\
Breast carcinoma & 2 \\
Malignant melanoma & 2 \\
Other & 6 \\
\hline
\end{tabular}



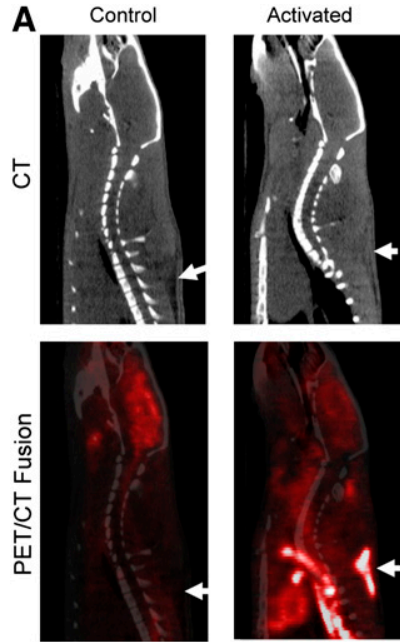

FIGURE 1. (A) Sagittal CT HU of subcutaneous paraspinal BAT (arrow) in rodent is greater under activated conditions than under control conditions. ${ }^{18} \mathrm{~F}-\mathrm{FDG}$ uptake of BAT is also increased under activated conditions. CT shows that BAT with increased fat density (right) fuses precisely to area of increased ${ }^{18} \mathrm{~F}-\mathrm{FDG}$ uptake. (B) CT image of resected BAT with surrounding WAT subjected to $4 \mathrm{~h}$ of cold-stimulation shows greater CT density that is closer to water (top) than control kept at room temperature (bottom). Under control conditions, BAT is difficult to distinguish from surrounding WAT. On CT, higher CT HUs are shown as areas of increased whiteness, whereas low HUs are increasingly black. Areas of WAT and BAT as seen on direct visual inspection are shown in schematic at right.

in BAT was defined as foci that had a $\mathrm{SUV}_{\max }$ greater than 3, whereas low uptake was defined as less than 2 . The $\mathrm{SUV}_{\max }$ was calculated on the basis of activity values in ROIs, which were manually placed on the maximum pixel area of the BAT (circular ROI with a minimum diameter of $5 \mathrm{~mm}$ corresponding to a 28 $\mathrm{mm}^{2}$ area; 2 ROIs per person) that displayed a $\mathrm{SUV}_{\max }$ higher than 3. ROIs placed on subcutaneous WAT on an axial slice at the hip level and in the liver served as controls (circular ROI with a diameter of $20 \mathrm{~mm}$ corresponding to a $340 \mathrm{~mm}^{2}$ area; 1 ROI in each area per person). The CT density of the focus with maximum
${ }^{18}$ F-FDG uptake was obtained using the ROI delineated on the PET image superimposed on the CT image and expressed in HUs.

\section{Statistical Analysis}

Results were expressed as mean values \pm SDs. The data from the cold-activated group were compared with those of the control group using the Mann-Whitney $U$ test. A paired $t$ test was used to compare the CT HUs of the 2 PET/CT scans (high and low ${ }^{18} \mathrm{~F}$ FDG uptake) within the same individual. A $P$ value of less than 0.05 was considered statistically significant.

\section{RESULTS}

\section{CT Appearance in BAT Tissue in Animals}

Figure 1 shows the differences in the micro-CT appearance in BAT between the 2 physical conditions (control and cold-activated) both in vivo (Fig. 1A) and ex vivo (Fig. 1B). The CT HUs of BAT were greater (whiter density) under the activated condition than under the control condition (Fig. 1A). ${ }^{18} \mathrm{~F}$-FDG uptake of BAT was also increased under the activated condition (fusion image). CT images of the excised BAT specimen (both conditions) displayed lower attenuation than water because of the large amount of subcutaneous fat tissue. CT HUs of activated BAT increased compared with the control BAT (Fig. 1B). The ${ }^{18}$ F-FDG uptake levels of BAT and CT HUs in rats are summarized in the upper part of Table 2. Significant differences were seen in the level of ${ }^{18} \mathrm{~F}$ FDG uptake and CT HU of BAT.

\section{Histopathologic Changes in BAT}

Figure 2 shows the microscopic findings of interscapular BAT with hematoxylin-eosin staining. Numerous lipid vacuoles were seen in the BAT cells of the control animals. In contrast, the size and number of lipid vacuoles was much smaller in the BAT of the cold-exposed animals. The total lipid content of BAT was significantly reduced after cold exposure.

\section{PET/CT Findings in Patients}

The ${ }^{18} \mathrm{~F}-\mathrm{FDG}$ uptake and CT HUs of patients are summarized in the lower part of Table 2. The CT HUs in scans with high SUV were significantly higher than the CT

\section{TABLE 2. Summary of In Vivo Imaging Studies}

\begin{tabular}{|c|c|c|c|}
\hline Study type & First parameter & Second parameter & $\boldsymbol{P}$ \\
\hline Rat & Cold-activated & Control & \\
\hline${ }^{18} \mathrm{~F}-\mathrm{FDG}$ uptake $\left(\mathrm{Bq} / \mathrm{cm}^{3}\right.$ of BAT) & $1,982.8 \pm 361.3$ & $892.9 \pm 357.7$ & $<0.05$ \\
\hline CT HU (BAT) & $-12.4 \pm 22.4$ & $-27.9 \pm 9.6$ & $<0.05$ \\
\hline CT HU (WAT) & $-69.3 \pm 9.7$ & $-75.6 \pm 9.0$ & NS \\
\hline CT HU (liver) & $19.8 \pm 4.2$ & $23.6 \pm 9.9$ & NS \\
\hline Human & High SUV & Low SUV & \\
\hline${ }^{18} \mathrm{~F}-\mathrm{FDG}$ uptake (SUV $\max$ of BAT) & $6.6 \pm 2.8$ & $0.9 \pm 0.5$ & $<0.05$ \\
\hline CT HU (BAT) & $-71.6 \pm 18.0$ & $-104.4 \pm 16.8$ & $<0.05$ \\
\hline CT HU (WAT) & $-101.8 \pm 6.4$ & $-102.6 \pm 5.1$ & NS \\
\hline CT HU (liver) & $56.0 \pm 7.7$ & $56.2 \pm 7.4$ & NS \\
\hline Frequency during cold season* & $19 / 23$ & $4 / 23$ & $<0.05$ \\
\hline
\end{tabular}




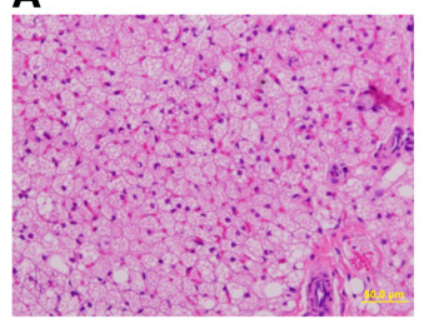

B

FIGURE 2. On light microscopy, major structural changes can be seen in BAT cells stained with hematoxylin-eosin. Many large lipid vacuoles are seen in control BAT cells (A), but vacuoles are almost nonexistent in BAT cells exposed to cold (B). Lipid content is much lower in cold-stimulated BAT.

HUs in the scans with low SUV $(-71.6 \pm 18.0$ vs. $-104.4 \pm$ 16.8; $P<0.05$ ) (Fig. 3A). When analyzed within the same individual, the CT HUs increased in the high-SUV scan in all but 2 foci in 2 different patients. There was a statistically significant difference in the CT HUs of BAT between the 2 conditions (paired $t$ test; $P<0.01$ ). On the other hand, no difference was seen in either the CT HUs of WAT or the liver in patients in the high and low BAT activity states (Table 2). When we plotted the CT HUs against the ${ }^{18} \mathrm{~F}-\mathrm{FDG}$ uptake in all 92 ROIs, there was a significant positive correlation between the 2 values $(R=$ 0.66, $P<0.01$ ) (Fig. 3B).

On visual inspection of the CT scans, some of the activated BAT appeared to be cloudy and was distinguishable from nonactivated BAT. Figure 4 shows a representative case.

\section{DISCUSSION}

We found that the CT HUs of BAT increased under activated conditions, as compared with the nonactivated conditions, in both the patients and the rat model. An increase in CT HUs was seen in all patients except two. It is possible their duration of BAT activation was brief, and perhaps there had not been pronounced lipid consumption.
In aggregate, these data indicate that the change in CT HUs was specific to BAT and related to the activation-induced physiologic alterations in this tissue.

In the animal model, the histology of BAT revealed that the total lipid content of BAT was substantially decreased under the 4-h cold-activated condition. Our data indicate that a decrease in lipid density in cold-activated BAT is likely responsible for the increase in the CT HUs. The somewhat higher CT HUs in the animal tissues may be related to resolution limitations for the small regions of BAT seen in animal imaging.

In humans, there was a strong positive correlation between the CT HUs and SUV $(r=0.66, P<0.01)$. The change in CT HUs and change in SUV were also positively correlated. These results indicate that the BAT CT HUs typically increase in parallel with the ${ }^{18} \mathrm{~F}$-FDG uptake level. This high BAT uptake was observed more often in colder months $(P=0.05)$. These results are compatible with previous reports $(1-3)$.

In the present study, the CT HUs of BAT in rats increased after cold-exposure in parallel with an increased uptake of ${ }^{18}$ F-FDG. As shown in Figure 2, this result can be partly attributed to a decrease in BAT lipid content. Although BAT derives much of its energy needs from glucose, it also can rapidly consume fat. Indeed, lipids of varying chain lengths have been shown to both facilitate uncoupling and serve as substrates for BAT metabolism (13). We believe rapid consumption of stored lipid is the predominant cause of the increase in HUs with BAT activation, with both glucose and lipid use occurring. A previous report has shown that activated BAT has a greater blood flow than nonactivated BAT (14), and increased blood flow and volume may also contribute to the rise in HUs of BAT.

In this study, we showed that CT HUs were increased by the activation of BAT. Because all CT HUs of BAT in our patient study were negative, one would be unlikely to misinterpret this uptake as occurring in anything other than lipid-containing tissue. However, fat tissue with modestly increased CT HUs may be indistinguishable from panniculitis or lipid-containing tumor tissue (15). Some reports have suggested that the ${ }^{18} \mathrm{~F}-\mathrm{FDG}$ uptake level of BAT in

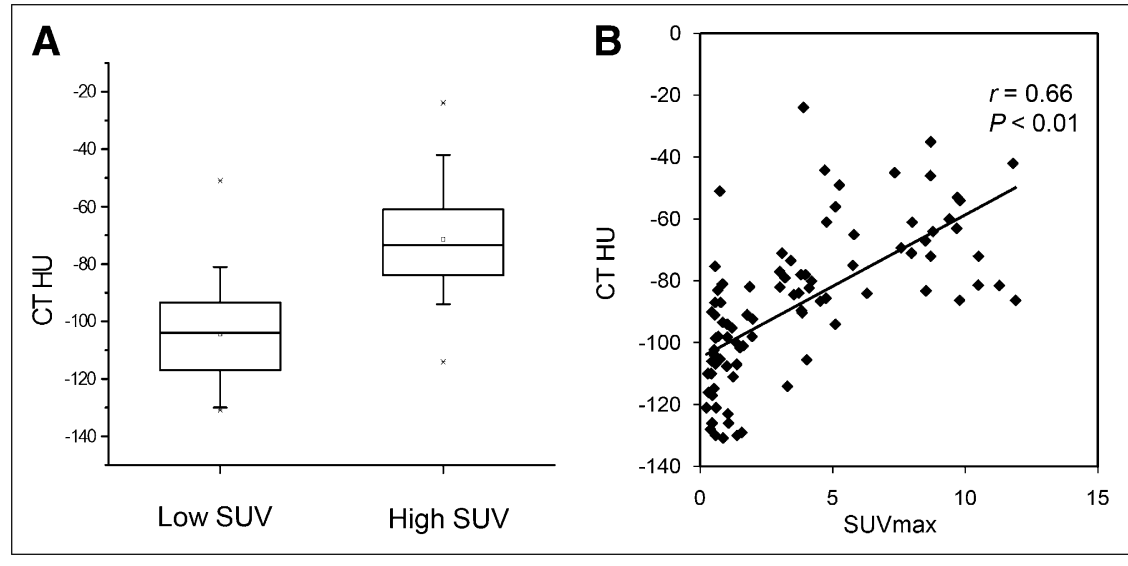

FIGURE 3. (A) Average CT HUs of BAT (46 regions from 23 patients) between high SUV for ${ }^{18} \mathrm{~F}-\mathrm{FDG}$ and low SUV for ${ }^{18} \mathrm{~F}-\mathrm{FDG}$ is shown. CT HUs in scans with high SUV were significantly higher than CT HUs in scans with low SUV $(-71.6 \pm 18.0$ vs. $-104.4 \pm 16.8 ; P<$ 0.05). (B) When we plotted CT HUs against ${ }^{18} \mathrm{~F}-\mathrm{FDG}$ uptake in all $92 \mathrm{ROIs}$ there was significant positive correlation between the 2 values $(R=0.66, P<$ 0.01). 
A
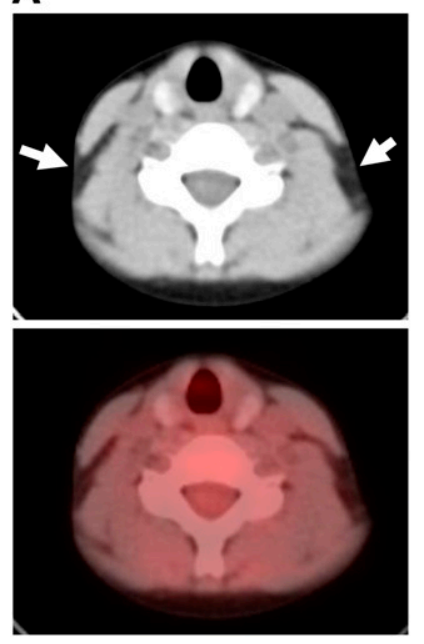

B
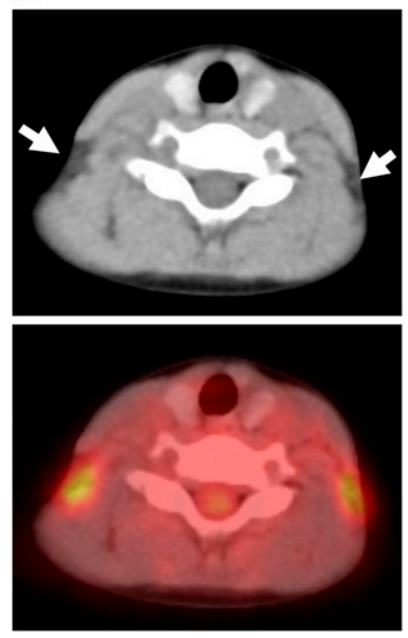

FIGURE 4. Representative study of patient in whom CT HU of BAT is increased in parallel with increase in ${ }^{18} \mathrm{~F}-\mathrm{FDG}$ uptake. A 20-y-old man with malignant lymphoma displayed bilateral cloudy BAT (increased CT HUs of -53 and -56 ) with avid ${ }^{18} \mathrm{~F}-\mathrm{FDG}$ uptake $\left(\mathrm{SUV}_{\max }=3.4\right.$ and 3.2) (B), whereas baseline scan showed low-attenuation BAT (CT HUs of -87 and -85$)$ with low ${ }^{18} \mathrm{~F}-\mathrm{FDG}$ uptake $\left(\mathrm{SUV}_{\max }=\right.$ 0.9 and 1.0) (A). Both CT images are displayed with same window level $(-20)$ and narrow window width (300) of CT HU to emphasize small difference of CT density in BAT.

patients with pheochromocytoma may be affected by the serum level of catecholamines $(16,17)$. Our findings suggest that direct measurement of CT HUs can help confirm fat density to be activated BAT. Although ${ }^{18}$ F-FDG PET appears robust at identifying BAT, CT characteristics may also prove useful and warrant further study as a tool to probe the presence of BAT and its volume and functional state. High ${ }^{18}$ F-FDG uptake in tissues in the expected location of BAT, with slightly higher CT HUs than in typical WAT, are likely due to BAT and should not be confused with disease.

\section{CONCLUSION}

The CT HUs of BAT are significantly greater under activated conditions for BAT than under nonactivated conditions in both patients and animal models. This change is likely due to lipid consumption by BAT. Higher CT HUs in BAT than WAT may occur and should not be confused with ${ }^{18} \mathrm{~F}-\mathrm{FDG}$-avid metastases.

\section{REFERENCES}

1. Astrup A, Bulow J, Madsen J, Christensen NJ. Contribution of BAT and skeletal muscle to thermogenesis induced by ephedrine in man. Am J Physiol. 1985; 248:E507-E515.

2. van Marken Lichtenbelt WD, Vanhommerig JW, Smulders NM, et al. Coldactivated brown adipose tissue in healthy men. N Engl J Med. 2009;360:15001508 .

3. Cohade C, Mourtzikos KA, Wahl RL. "USA-Fat": prevalence is related to ambient outdoor temperature-evaluation with ${ }^{18}$ F-FDG PET/CT. J Nucl Med. 2003; 44:1267-1270.

4. Cohade C, Osman M, Pannu HK, Wahl RL. Uptake in supraclavicular area fat ("USA-Fat"): description on ${ }^{18}$ F-FDG PET/CT. J Nucl Med. 2003;44:170-176.

5. Hany TF, Gharehpapagh E, Kamel EM, Buck A, Himms-Hagen J, von Schulthess GK. Brown adipose tissue: a factor to consider in symmetrical tracer uptake in the neck and upper chest region. Eur J Nucl Med Mol Imaging. 2002;29:1393-1398.

6. Cypess AM, Lehman S, Williams G, et al. Identification and importance of brown adipose tissue in adult humans. $N$ Engl J Med. 2009;360:1509-1517.

7. Clarke JR, Brglevska S, Lau EW, Ramdave S, Hicks RJ. Atypical brown fat distribution in young males demonstrated on PET/CT. Clin Nucl Med. 2007; 32:679-682.

8. Hyun IY, Kim SG. FDG uptake in parahepatic brown fat mimics peritoneal carcinomatosis in a malignant ovarian germ cell tumor: resolution with temperature control. Clin Nucl Med. 2008;33:799-801.

9. Tatsumi M, Engles JM, Ishimori T, Nicely O, Cohade C, Wahl RL. Intense ${ }^{18} \mathrm{~F}-$ FDG uptake in brown fat can be reduced pharmacologically. J Nucl Med. 2004; 45:1189-1193.

10. Virtanen KA, Lidell ME, Orava J, et al. Functional brown adipose tissue in healthy adults. $N$ Engl J Med. 2009;360:1518-1525.

11. Christensen CR, Clark PB, Morton KA. Reversal of hypermetabolic brown adipose tissue in F-18 FDG PET imaging. Clin Nucl Med. 2006;31:193-196.

12. Baba S, Engles JM, Huso DL, Ishimori T, Wahl RL. Comparison of uptake of multiple clinical radiotracers into brown adipose tissue under cold-stimulated and nonstimulated conditions. J Nucl Med. 2007;48:1715-1723.

13. Shabalina IG, Backlund EC, Bar-Tana J, Cannon B, Nedergaard J. Within brown-fat cells, UCP1-mediated fatty acid-induced uncoupling is independent of fatty acid metabolism. Biochim Biophys Acta. 2008;1777:642-650.

14. Astrup A, Bulow J, Madsen J. Interscapular brown adipose tissue blood flow in the rat: determination with ${ }^{133}$ xenon clearance compared to the microsphere method. Pflugers Arch. 1984;401:414-417.

15. Smith CS, Teruya-Feldstein J, Caravelli JF, Yeung HW. False-positive findings on ${ }^{18}$ F-FDG PET/CT: differentiation of hibernoma and malignant fatty tumor on the basis of fluctuating standardized uptake values. AJR. 2008;190:1091-1096.

16. Yamaga LY, Thom AF, Wagner J, Baroni RH, Hidal JT, Funari MG. The effect of catecholamines on the glucose uptake in brown adipose tissue demonstrated by ${ }^{18} \mathrm{~F}-\mathrm{FDG}$ PET/CT in a patient with adrenal pheochromocytoma. Eur J Nucl Med Mol Imaging. 2008;35:446-447.

17. Kuji I, Imabayashi E, Minagawa A, Matsuda H, Miyauchi T. Brown adipose tissue demonstrating intense FDG uptake in a patient with mediastinal pheochromocytoma. Ann Nucl Med. 2008;22:231-235. 